\title{
The Evaluation of Aerodynamic Interaction of Wind Blade Using Fluid Structure Interaction Method
}

\author{
Kyoungsoo Lee, Ziaul Huque, Raghava Kommalapati, and Sang-Eul Han
}

\begin{abstract}
This paper presents the evaluation process of aerodynamic load on wind turbine blade using Fluid Structure Interaction (FSI) method. The aerodynamic loads are determined using 3D Computational Fluid Dynamics (CFD) and are applied to the wind blade structure with the help of an interface which relates the FSI interaction. The interface shares the identical surface topology. The steady and transient CFD analyses with k- SST turbulence model are performed for 1-way and 2-way interaction, which are defined by using the interface between fluid and structure domains. A parameterized 3D CAD models for National Renewable Energy Laboratory (NREL) Phase VI wind blade are used for the analyses. The verification of CFD results are performed by comparing with experimental data, which were obtained from NREL. The calculated and verified aerodynamic loads are applied to the wind blade structural model. The effective structural stiffness was derived from the frequency based eigen-value analysis. The static and time dependent transient structural analyses were performed. From the mesh-sensitivity parametric studies, the wind power coefficient or torque value is found to be strongly affected by the fluid mesh characteristics. The structural mesh characteristic was important in the mapping process of CFD aerodynamic load to the structure.
\end{abstract}

Index Terms - Wind energy, wind blade, FSI, CFD, NREL Phase VI, airfoil.

\section{INTRODUCTION}

The design of wind blade is highly dependent on the evaluation of aerodynamic wind load. To increase the wind turbine power coefficient, it should extract more wind kinetic energy. Hence the understanding of the aerodynamics around the blade is that much important to obtain the optimum wind blade shape.

There are well known approaches to calculate the aerodynamic load of wind blade. Wind tunnel tests are considered as the most exact and practical method and are sought for validation by researchers. However, it needs comprehensive equipments and knowledge to perform the test.

Manuscript received March 1, 2014; revised July 2, 2014. This research was supported by the National Science Foundation (NSF) through the Center for Energy and Environmental Sustainability (CEES), a CREST Center (Award No. 1036593)

Kyoungsoo Lee is with Center for Energy and Environmental Sustainability (CEES), Prairie View A\&M University, Prairie View, TX, 77446, USA (e-mail: kylee@pvamu.edu).

Ziaul Huque is with the Mechanical Engineering Department, CEES, Prairie View A\&M University, Prairie View, TX, 77446, USA (e-mail: zihuque@pvamu.edu).

Raghava Kommalapati is with the Civil \& Environmental Engineering Department, CEES, Prairie View A\&M University, Prairie View, TX, 77446 , USA (e-mail: rrkommalapati@pvamu.edu).

Sang-Eul Han is with the School of Architecture, Inha University, Incheon, South Korea (e-mail: hsang@inha.ac.kr).
The researchers can utilize the publicly reported 2D airfoil wind tunnel test results to design their wind blade. The practical and more important data of $3 \mathrm{D}$ wind blade wind tunnel test is very difficult to obtain and rarely available. Moreover, the wind blade is highly shape sensitive especially for the twist angle and the varying span-wise sectional shape. The 2D airfoil aerodynamic coefficients such as lift and drag, with various angles of attack do not correspond to the 3D results. The idea of using $2 \mathrm{D}$ wind tunnel test data for $3 \mathrm{D}$ simulation and design is not desirable.

The alternatives are to use computational methods like Blade Element Momentum method (BEM) [1], Vortex method (VM) [2] and Computational Fluid Dynamics (CFD) [3]-[8] to calculate the aerodynamic load of 3D wind blade. However, BEM and VM are basically dependent on the accuracy of wind tunnel test data until now.

Even though, CFD is known to be very difficult to apply in the unsteady aerodynamic phenomenon, it can calculate the sectional airfoil aerodynamic coefficient independently using high end computational software and hardware with comprehensive turbulence models. Verifications of CFD results are necessary though before applying to practical projects. Also, the surface pressure obtained from CFD can be directly applied as the structural load using an interface to connect fluid flow and structural analyses called as Fluid Structure Interaction (FSI) [9].

In this study, we introduce a process of FSI where results from CFD are directly applied to structural analyses and vice versa with the use of an appropriate interface. The interface between the two physical domains can transfer the mutually needed data. Through this systematic FSI analysis process, the aerodynamic effects of the fluid domain on the structure can be easily seen. The structural analysis and design are performed through the interface by explicitly loading the aerodynamic loads to the FEM structural model automatically without calculating any sectional aerodynamic coefficients.

\section{AERODYNAMIC OF WIND BLADE AND WIND TUNNEL}

The wind loading is one of the most general external loading conditions which any structure will experience during their service time. Usually these structures are designed to minimize the aerodynamic wind load. These works are geared towards saving structural material and produce optimized economical structural design. In contrast to general structural system, the wind blade is designed to maximize the aerodynamic load. A turbine system is a mechanically rigid multi-body system which converts the wind energy to rotating torque energy. In order to produce maximum efficiency, large-scale structural systems of wind turbine with tower and 
base foundation are needed. The fundamental power generation and control system are also essential. The complexities and difficulties come from these highly technical assemblies. Among all the components of a wind turbine system, the shape sensitive rotating wind blade is the most important part to increase the power generation rate (power coefficient).

A wind blade shares the aerodynamic theory with airplane wing and helicopter blade. These systems utilize the higher lifting force to rotate and to lift. The airplane wing stays stationary when it is in operating condition. The wind blade is closer to the rotating helicopter blade. However, the high speed rotating helicopter blade should be thin and should have small angle of attack. As a result, there are little unsteady turbulent phenomenon compared with wind blade system.

To determine the optimized wind blade shape which generates the maximum power in low operating inflow wind speed, we need to evaluate the aerodynamic force coefficient which will rotate the wind blade about primary axis. Airplane wing has constant sectional airfoil shape along the span-wise dimension because of nearly constant angle of attack around it. However, the wind blade rotates in induced angular velocity and hence it has linearly varying rotating velocities. The calculated angles of attack along the span are shown to have nonlinear values. The wind blade may also have nonlinear twist angle to minimize the angle of attack and turbulence around the blade wall along the span.

Since the blade is rotating and has nonlinear twist angle, the surface stream on the suction surface flows toward the direction of the tip from the root side. Because of the reported 2D airfoil wind tunnel test data for various angles of attack are shown to have noticeable errors compared with 3D wind tunnel test data, the wind blade cannot be designed based on 2D aerodynamic theory and design codes. These codes are based on 2D wind tunnel tests and 2D BEM formulations. Comprehensive 3D aerodynamic data should be taken into account in wind blade design process. To meet these requirements, the real scale $3 \mathrm{D}$ wind tunnel studies are essential. The NREL Phase VI wind blade is a well known publicly accessible 3D wind tunnel test project. From these rigorous experimental wind tunnel tests, accurate quantitative aerodynamic and structural measurements on a full scale wind turbine blades were performed to provide the needs of accurate $3 \mathrm{D}$ aerodynamic forces which takes into account the inflow turbulence and shear across the rotor plane.

BEM is frequently preferred in wind blade design code because of its simplicity and overall accuracy. However, it depends on the aerodynamic force coefficients from 2D or 3D wind tunnel test data and has limitation only for asymmetric cases. It shows difficulties when taking into account the unsteady and nonlinear transient rotor yaw and rotating wake flow effects like any other numerical methods. VM is an ongoing active research area, which considers the 3D unsteady flow effects. Even though they have appealing numeric advantages, the aerodynamic coefficient of $3 \mathrm{D}$ wind tunnel test data should be used for evaluating the accuracy of aerodynamic analysis and application. Even though CFD has a similar difficulties and limitations, it can easily be applied to the change of shapes of wind blade around a reference shape.

\section{CFD FOR FLUID STRUCTURE INTERACTION}

The wind loading is one of the most general external loading conditions which any structure will experience during their service time. Usually these structures are designed to minimize the aerodynamic wind load. These works are geared towards saving structural material and produce optimized economical structural design. In contrast to general structural system, the wind blade is designed to maximize the aerodynamic load. A turbine system is a mechanically rigid multi-body system which converts the wind energy to rotating torque energy. In order to produce maximum efficiency, large-scale structural systems of wind turbine with tower and base foundation are needed. The fundamental power generation and control system are also essential. The complexities and difficulties come from these highly technical assemblies. Among all the components of a wind turbine system, the shape sensitive rotating wind blade is the most important part to increase the power generation rate (power coefficient).

\section{CONCEPT OF FLUID STRUCTURE INTERACTION}

The FSI are used in wide range of multi-physical problem in which the fluid effects are dominant but difficult to evaluate for structural part. Especially high curvature free shape of 3D wind blade is a challenging problem to apply on the FSI simulation, because of its difficulty in evaluating the aerodynamic force of the surface.

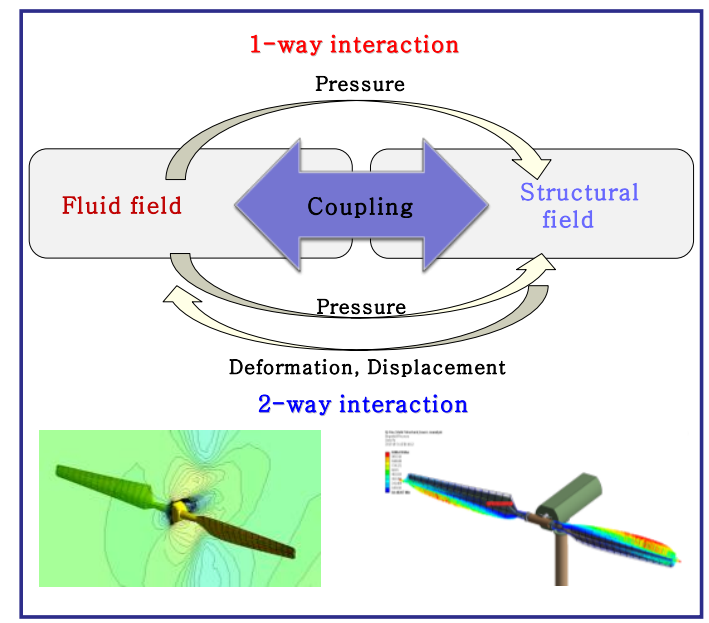

Fig. 1. Concept of fluid structure interaction.

The fundamental pre-requirements for FSI are the verification of pressure loading value from CFD and the definition of interface between fluid and structural domain. As shown in Fig. 1, the interface transfers data from fluid to structure or structure to fluid. These interactions are called as 1-way. If the interface transfers the data to both sides sequentially, we call it as 2-way. Fluid domain transfers the pressure or temperature to the structure, and structure transfers usually the displacement to the fluid. From these interactions, the fluid domain updates the domain mesh from structural deformation. The structural part deforms due to the pressure or temperature load from the fluid domain. Unlike the BEM or VM methods, the structural part can be modeled using FEM procedure. The response of the structure relies on 
the material and solid modeling quality. The surface of structural part should share identical topology with CFD domain.

The rotating wind blade are vibrating with its frequency to the direction of first eigen-mode shape which is independent to the wind flow direction but dependent to the local axis. The vibrating frequency of wind blade is dependent on the stiffness and mass of blade. If the tower is considered, the vibrating model of tower will be coupled with blade. Lift causes bending in the flap-wise direction while air flow around the blade cause edge-wise bending. Flap-wise bending involves tension on the pressure (upwind) side and compression on the suction (downwind) side. Edgewise bending involves tension on the leading edge and compression on the trailing edge. Hence, 3D wind blade may experience the unsteady transient of fluctuation and vortex shedding. CFD is one of the options to capture these unsteady effects, even though it has numerical difficulties and limitations. To consider the time dependent unsteady effect of fluctuation and vibration in numerical method, the fluid domain must be changed along with structural vibration in transient analysis. Hence, to simulate these problems, the interface should take into account the interaction of both sides using 2-way FSI. The 1-way FSI is used for steady static problem and 2-way is for unsteady transient problem.

\section{FSI SIMULATION FOR NREL PHASE VI WIND BLADE}

The comprehensive information about the unsteady aerodynamic experimental data for NREL Phase VI wind turbine blade (Fig. 2 and Fig. 3) is publicly accessible [10]-[13]. Providing full scale, 3D aerodynamic behavior for Horizontal-axis wind blade (HAWT) was the primary objective of this work. The wind turbine and wind blade undergo complex aerodynamic phenomena in field operation [12], [13]. This phenomenon is not considered in wind turbine design codes which are derived from the steady state $2 \mathrm{D}$ wind tunnel airfoil test. To provide the needs of accurate 3D aerodynamic force which considers the inflow turbulence and shear across the rotor plane, the wind tunnel test was performed by NREL using Phase VI wind blade [12], [13]. From the rigorous experimental wind tunnel tests, accurate quantitative aerodynamic and structural measurements on a full scale wind turbine blades were performed. The wind tunnel test data can be used for reference values. There are number of research works which were reported to show the accuracy and applicability of CFD for 3D wind blade [4]-[8].

To meet the purpose of this paper, the FSI simulations were performed. The fluid domain aerodynamic effects are tested by using CFD and shows excellent agreement with reference values of NREL wind tunnel test [4]-[8], [12], [13]. The overall numerical procedures were performed using commercial software Ansys. The fluid field domain aerodynamic loads are determined by using Ansys-CFX and those are transferred to the wind blade structure by the FSI interaction. The interface shares the identical surface topology. The steady and transient CFD analyses are performed for 1-way and 2-way interaction.

As shown in Fig. 4 and Fig. 5, a parameterized 3D CAD model and fluid domain meshes for NREL Phase VI [12] are used for the CFD and FSI analyses. The calculated aerodynamic loads are applied to the wind blade structural model which was developed approximately according to the reported structural information [12]. Fig. 6 shows the eigen-mode shapes of structural model to estimate and verify the stiffness and mass quantity of it. The assembled overall structure comprised of wind blade, shaft, nacelle and tower. The effective structural stiffness was derived from the frequency based eigen-value analysis and shows good agreement with experimental data [12] for natural frequency.

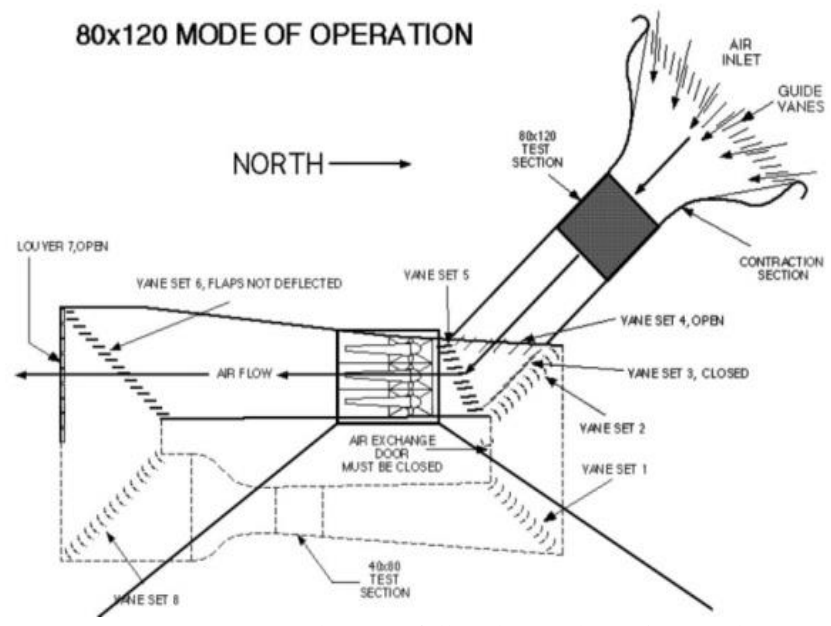

(a) NASA Ames research centre full scale aerodynamic complex.

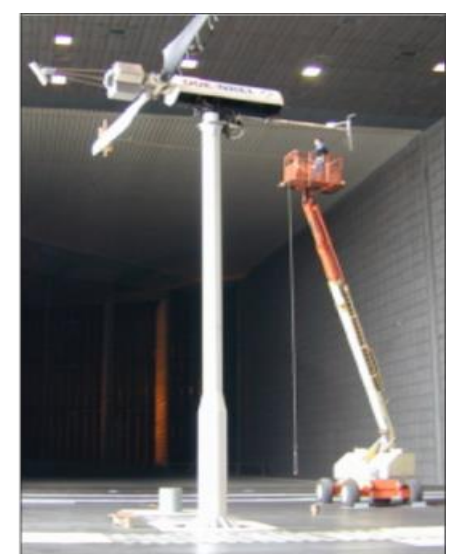

(b) Full scale NREL Phase VI wind turbine blade.

Fig. 2. NASA Ames research center full scale aerodynamic complex : NREL Phase VI wind turbine blade [12],[13].

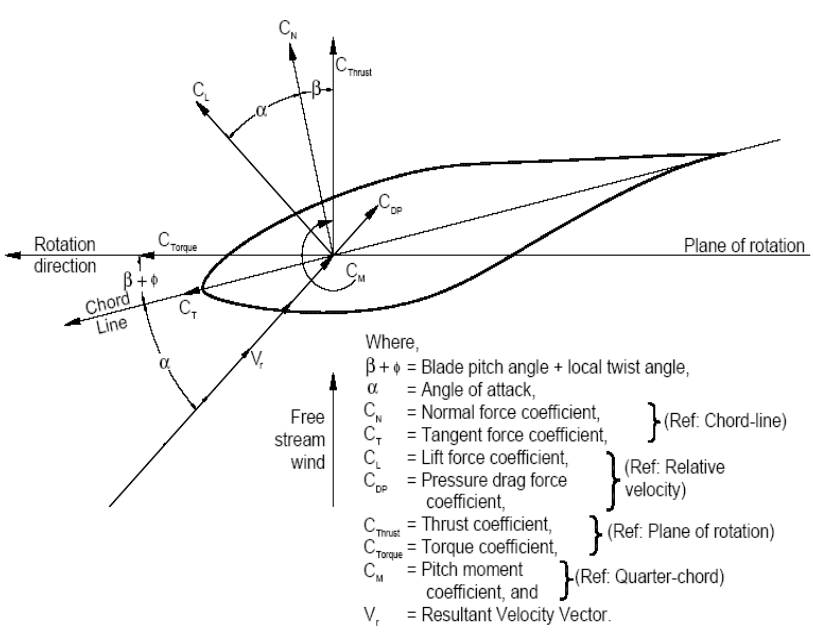

(a) Aerodynamic force coefficient conventions. 


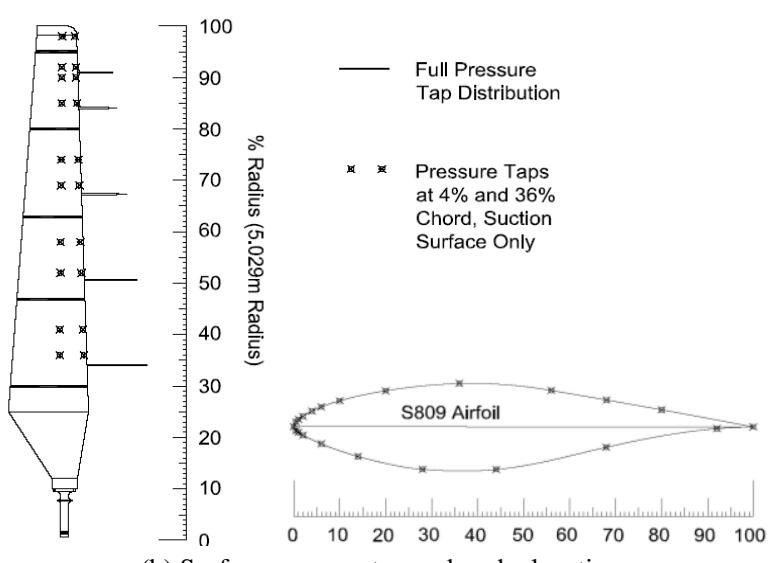

(b) Surface pressure tap and probe locations.

Fig. 3. Surface pressure tap location and aerodynamic force coefficient conventions: NREL Phase VI wind blade [12], [13]

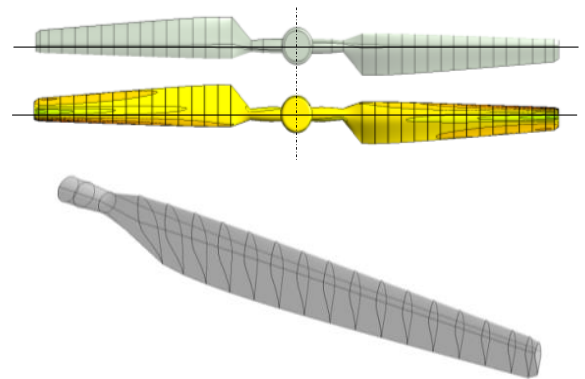

(a) NREL Phase VI wind blade.

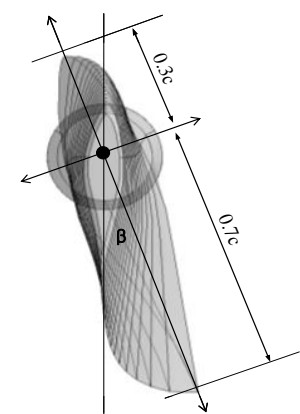

(b) Twist angel section.

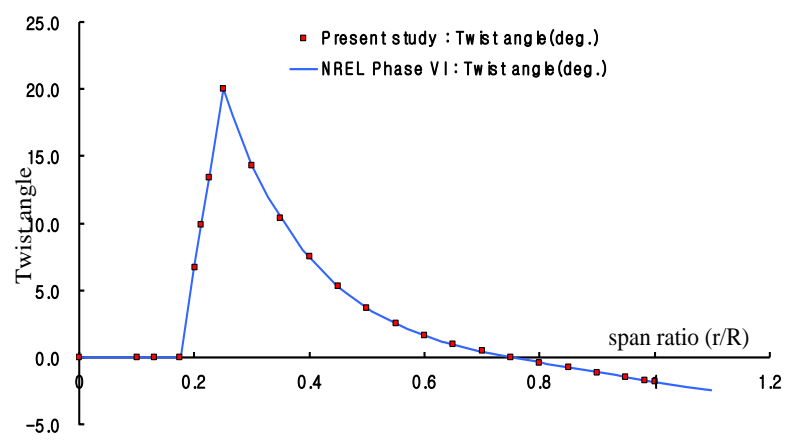

(c) Twist angle distribution along span ratio.

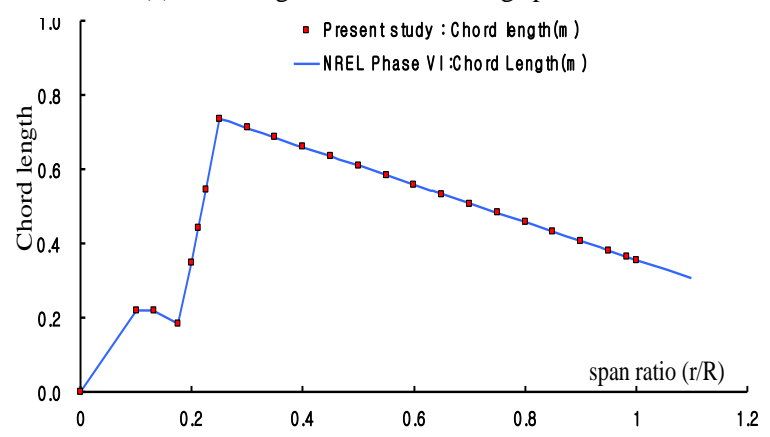

(d) Taper (chord length) distribution along span ratio. Fig. 4. NREL Phase VI wind blade model for CFD.

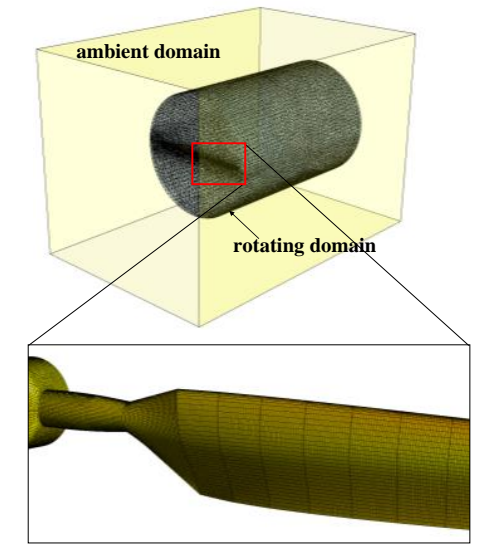

Fig. 5. Fluid domain and blade mesh representation.

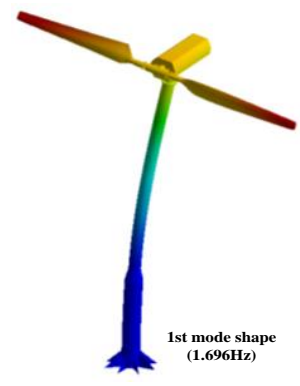

(a) 1st mode shape

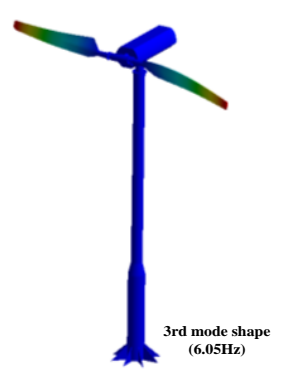

(c) 3rd mode shape

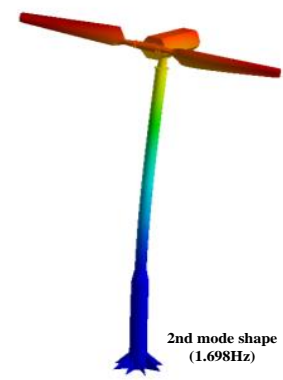

(b) 2nd mode shape

Fig. 7 and Fig. 8 show the 1-way and 2-way FSI simulation process respectively. The verified CFD results are used for wind blade pressure load and transferred to the structural surface load. Only the interaction of wind blade surface considered to verify the wind blade aerodynamic load. The static and transient structural responses were tested using 1-way and 2-way FSI respectively. In 2-way FSI simulation, as shown in Fig. 8(a) simultaneous interactive calculating processes are performed to match the interface domain. Hence, the FSI simulation is strongly related to each other in 2-way case.

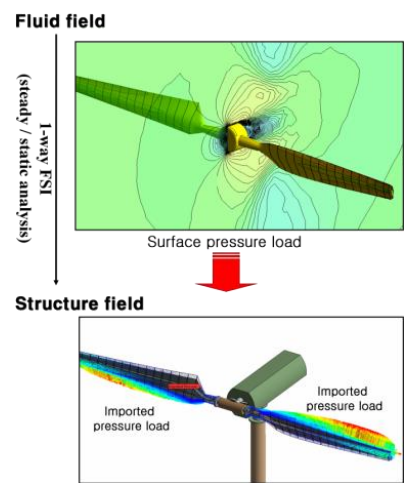

Fig. 7. 1-way FSI process: steady state fluid/static structure. 
2-way FSI Fluid field $\stackrel{\text { (unsteady / transient analysis) }}{\longrightarrow}$ Structure field

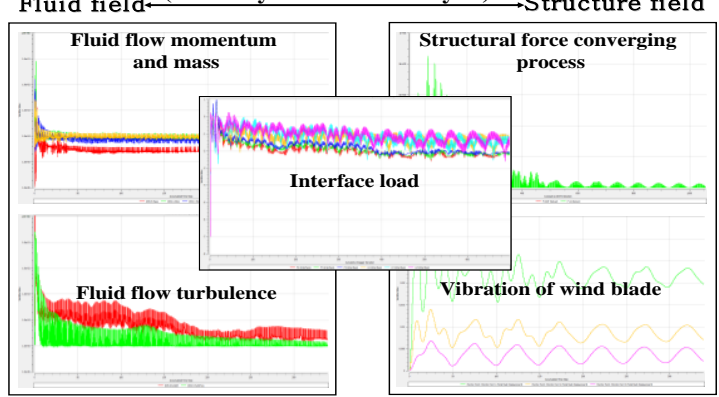

(a) 2-way FSI process.

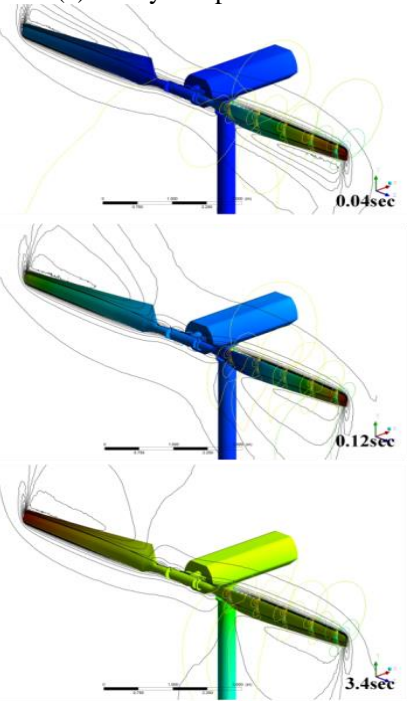

(b) Isometric view.

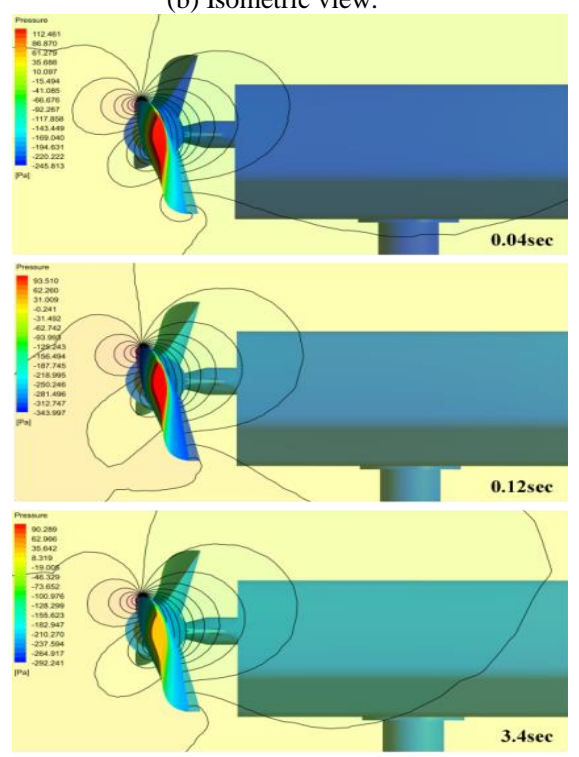

(c) Side view.

Fig. 8. 2-way FSI results :pressure and velocity contour.

Only $7 \mathrm{~m} / \mathrm{s}$ wind speed case is considered with $72 \mathrm{rpm}$ rotating velocity to show the numerical characteristics of this study. From the mesh-sensitivity parametric studies, the wind power coefficient or torque value of wind blade is found to be highly dependent on the fluid mesh characteristics. The static structural responses using 1-way FSI are found to be in good agreement with the results obtained by using sectional aerodynamic force which is based on the BEM formulation. The supporting tower has little effect on the overall stability but it increases the displacement for static and transient cases. The time dependent vibration of wind blade shows the mode composition of natural frequency. However, the fundamental vibrating motion of wind turbine is dominant to that of tower not wind blade because of lightweight nature of wind blade.

\section{CONCLUSIONS}

The evaluation of aerodynamic interaction of NFEL Phase VI wind blade using FSI is presented. The CFD results are shown to be in excellent agreement with wind tunnel test data using k- $\omega$ SST turbulence model. The assumed structural FEM model produced similar natural frequency on the experiment. From the 1-way and 2-way FSI simulation for static and transient analysis respectively, the dominant vibrating motion of overall turbine blade is not blade but tower, and the time dependent transient vibrating motion of wind blade finally converged to the static motion. The FSI interface transferred the surface pressure to the structure and structural displacement to the fluid accurately and successfully. The wind power coefficient of wind blade is highly affected by the fluid mesh quality. The structural mesh characteristic was important in mapping process of CFD aerodynamic load to the structure.

\section{REFERENCES}

[1] L. I. Lago, F. L. Ponta, and A. D. Otero, "Analysis of alternative adaptive geometrical configurations for the NREL-5 MW wind turbine blade," Renewable Energy, vol. 59, pp. 13-22, 2013.

[2] H. Kim, S. Lee, E. Son, S. Lee, and S. Lee, "Aerodynamic noise analysis of large horizontal axis wind turbines considering fluid structure interaction," Renewable Energy, vol. 42, pp. 46-53, 2012.

[3] E. P. N. Duque, M. D. Burklund, and W. Johnson, "Navier-Stokes and comprehensive analysis performance predictions of the NREL phase VI experiment," Journal of Solar Energy Engineering, vol. 125, pp. 457-467, 2003.

[4] C. Tongchitpakdee, S. Benjanirat, and L. N. Sankar, "Numerical simulation of the aerodynamics of horizontal axis wind turbines under yawed flow conditions," in Proc. 43th AIAA Aerospace Sciences Meeting and Exhibit, Reno, NV, January 2005, AIAA, 2005, pp. 281-304.

[5] N. Sezer-Uzol and N. L. Long, "3-D time-accurate CFD simulations of wind turbine rotor flow fields," in Proc. 44th AIAA Aerospace Sciences Meeting and Exhibit, Reno, NV, January 2006, AIAA, 2006, pp. 1-23.

[6] M. M. Yelmule and E. Anjuri, "CFD predictions of NREL phase VI rotor experiments in NASA/AMES wind tunnel," International Journal of Renewable Energy Research, vol. 3, pp. 250-260, 2013.

[7] M. A. Hossain, Z. Huque, R. R. Kommalapati, and S. Khan, "Numeric investigation of compressible flow over NREL phase VI airfoil," International Journal of Engineering Research \& Technology, vol. 2, pp. 1-6, 2013.

[8] M. A. Hossain, Z. Huque, and R. R. Kommalapati, "Propagation of shock on NREL phase VI wind turbine airfoil under compressible flow," Journal of Renewable Energy, vol. 2013, 2013.

[9] Y. Bazilevs, M. C. Hsua, and M. A. Scottb, "Isogeometric fluid-structure interaction analysis with emphasis on non-matching discretizations and with application to wind turbines," Computer Methods in Applied Mechanics and Engineering, vol. 249-252, pp. 28-41, 2012.

[10] D. M. Sommers and J. Tangler, Design and Experimental Results for the S809 Airfoil, National Renewable Energy Laboratory (NREL), 1997.

[11] D. M. Sommers, "Design and experimental results for the s809 airfoil," Tech. rep., National Renewable Energy Laboratory (NREL), 1997.

[12] M. Hand, D. Simms, L. J. Fingersch, D. Jager, S. Larwood, J. Cotrell, and S. Schreck, "Unsteady aerodynamics experiment phase VI: Wind tunnel test configurations and available data campaigns," Tech. rep., National Renewable Energy Laboratory (NREL), 2001.

[13] S. Schreck, M. Hand, and L. J. Fingersch, "NREL unsteady aerodynamics experiment in the NASA-Ames wind tunnel: A comparison of predictions to measurements," Tech. rep., National Renewable Energy Laboratory (NREL), 2001. 


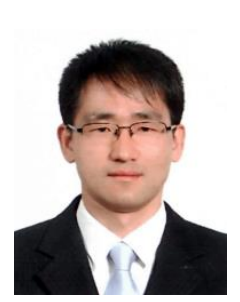

Kyoungsoo Lee received his BS, MS, Ph.D. degrees in Department of Architectural Engineering from Inha University, Incheon, South Korea. He is working for the CEES, Prairie View A\&M University, Prairie View, Texas, USA as a post doctor researcher. He was a research professor in Department of Civil \& Environmental Engineering, KAIST in South Korea. His professional areas are the structural engineering and design, CFD, FSI and impact \& blast simulation. Currently, he is focusing on the developing the sound noise simulation for the wind blade. Dr. Lee is a member of AIK, KSSC in South Korea.

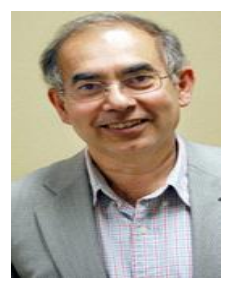

Ziaul Huque received his BS degree in mechanical engineering from Bangladesh University of Engineering and Technology, Bangladesh, MS degree in mechanical engineering from Clemson University, USA and Ph.D. degree in mechanical engineering from Oregon State University, USA. He is currently a professor in the Department of Mechanical Engineering and the director of Computational Fluid Dynamics Institute at Prairie View A\&M University. Professor Huque published over 50 journal and conference papers. His current research interests are wind turbine noise reduction, fluid-structure interaction, propulsion, inlet-ejector system of rocket based combined cycle engines, clean coal technology, self-propagating high-temperature synthesis. He received several excellences in teaching and service awards from Roy G. Perry College of Engineering, Lockheed-Martin Tactical Aircraft Systems Teaching Excellence Award, Welliver Summer Faculty Fellowship from Boeing in 2002 and NASA Summer Faculty Fellowship in 2003.

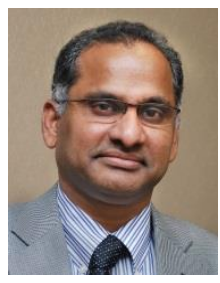

Raghava R. Kommalapati received MS and $\mathrm{PhD}$ degrees in civil (environmental engineering) from Louisiana State University, Baton rouge, LA, USA in 1994 and 1995 respectively. Prior to that he received his B.Tech degree in civil engineering and M.Tech degree in engineering structures from India. His major field of study is environmental engineering with particular focus on energy and environmental sustainability and air quality.

$\mathrm{He}$ is the director for Center for Energy and Environmental Sustainability and professor of civil \& environmental engineering at Prairie View A\&M
University, Prairie view, TX which is a part of the Texas A\&M University System. He is editor of 1 book, and published more than 30 peer-reviewed journal articles and more than 70 proceedings and presentations at regional, national and international conferences. Dr. Kommalapati's research interests include remediation of contaminated soils, industrial waste separations, air quality atmospheric air-fog interactions and environmental impacts of energy technologies.

Dr. Kommalapati is a licensed professional engineer (PE) in the state of Texas and a Board Certified Environmental Engineer (BCEE). He is a member of several professional organizations including, AEESP, AAEES, ACS, ASCE, ASEE and Honor societies of Tau Beta Phi, Phi Kappa Phi and Sigma Xi. He is an editorial board member of Journal of Studies in Atmospheric Science (SIAS), Current Advances in Environmental Science, International Open Journal of Energy Engineering, International Open Journal of Renewable \& Sustainable Energy, The Scientific World Journal, Environmental Chemistry Division, and Journal of Environmental Protection.

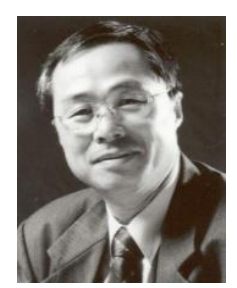

Sang-Eul Han was born in Hwasung, South Korea. He received his $\mathrm{Ph} . \mathrm{D}$. degree in architectural engineering form Kyoto University, Kyoto, Japan, 1994. He is a professor in School of Architecture, Inha University, Incheon, Korea from 1995 to current days. Dr. Han has published over 100 technical articles. His main research areas are nonlinear post buckling, shape finding, CFD, FSI and impact \&blast simulation. He is working for vibration control optimization system for tall building structure. Dr. Han is a member of IACSIT, AIK, COSEIK, KSSC, WEIK in South Korea 

Ocean Energy and Bioenergy 
